\title{
Incorporación de Generación Renovable y Segmentación de Redes Eléctricas
}

\section{Incorporation of Renewable Generation and Segmentation of Electric Networks}

Presentación: 6/10/2020

\section{Doctorando:}

\section{Carlos Bonetti}

Laboratorio de Métodos y Simulaciones Computacionales, Facultad Regional Rafaela, Universidad Tecnológica Nacional - Argentina. carlosbonetti3@gmail.com

\section{Director:}

\section{Gabriel Puccini}

\section{Co-director:}

\section{Jorge Vega}

\section{Resumen}

En el presente trabajo se desarrolla una metodología que facilita la transición de una red eléctrica centralizada tradicional a una configuración de red eléctrica basada en microredes. Se incorporan generadores inyectando potencia en posiciones estratégicas de la red. Además, partiendo de la topología de una red radial centralizada, se delimitan microredes con el propósito de otorgarles la mayor capacidad para que puedan funcionar de forma aislada. Esta optimización se realiza considerando la minimización de las pérdidas de la red y maximizando la autonomía potencial de las microredes obtenidas. Para desarrollar la metodología propuesta, se emplea recocido simulado como algoritmo metaheurístico de optimización.

Palabras clave: Microredes, Inyección de potencia, Minimización de Pérdidas, Recocido Simulado.

\begin{abstract}
In the present work, a methodology is developed that facilitates the transition from a traditional centralized electrical network to an electrical network configuration based on microgrids. Generators are incorporated by injecting power into strategic positions of the network. In addition, starting from the topology of a centralized radial network, microgrids are delimited with the purpose of granting them the greatest capability to work in isolation. This optimization is implemented considering the minimization of network losses and maximization of the potential autonomy of the obtained microgrids. To develop the proposed methodology, simulated annealing is used as a metaheuristic optimization algorithm.
\end{abstract}

Keywords: Microgrids, Power Injection, Loss Minimization, Simulated Annealing. 


\section{Introducción}

Actualmente se han logrado avances significativos en diversas formas de generar energía eléctrica. Una estrategia que permite maximizar el potencial beneficio de los recursos energéticos renovables consiste en instalar las fuentes de energía en posiciones estratégicas de una red eléctrica. Este nuevo escenario, denominado generación distribuida, busca minimizar las pérdidas instalando los generadores en puntos próximos a los centros de consumo (Rau, 1994; Atwa, 2010; Chowdhury, 2009). Además, en respuesta a la necesidad de una mayor eficiencia energética y confiabilidad, se desarrolló un nuevo concepto de red eléctrica denominado microred (Prommee, 2011). Las microredes son pequeñas redes eléctricas que operan normalmente conectadas a la red de distribución, pero pueden desconectarse y funcionar autónomamente. Esta gestión autónoma mejora la calidad del servicio eléctrico (Beyza, 2018).

Para diseñar microredes a partir de una red eléctrica conectada a un sistema de distribución centralizado, se presenta la necesidad de aprovechar las instalaciones existentes. El presente trabajo parte de una red eléctrica y plantea delimitarla para diseñar las microredes considerando la incorporación de generadores. Se busca determinar cuáles son las líneas más adecuadas para realizar una segmentación de modo que la red original adopte la configuración de varias microredes (Arefifar, 2012). Además, se busca establecer en qué puntos es conveniente instalar los generadores, considerando la autonomía potencial de las microredes diseñadas y la minimización de las pérdidas de la red. Así, la formulación del problema se realiza teniendo en cuenta dos objetivos: máxima autonomía y mínimas pérdidas. La naturaleza combinatoria del problema de tipo NP-completo demanda la búsqueda de soluciones mediante el uso de técnicas de optimización heurística. En el presente trabajo se implementó el algoritmo de búsqueda denominado recocido simulado.

\section{Desarrollo}

El diseño óptimo multiobjetivo consiste en encontrar la solución de compromiso entre dos objetivos contrapuestos. Un objetivo busca minimizar las pérdidas de la red, mientras que el otro busca minimizar el flujo de potencia en las líneas a segmentar de modo de formar microredes con máxima autonomía. La función objetivo del problema está dada por:

$$
f=\lambda \sum_{l=1}^{L} \operatorname{Per}_{l}+(1-\lambda) \sum_{l s=1}^{L S}\left|F P_{l s}\right|
$$

donde $L$ es el número total de líneas, $P e r_{l}$ es la pérdida en cada líneal, $L S$ es el total de líneas segmentadas, $F P_{l s}$ es el flujo de potencia en cada línea segmentada $l s$, y $\lambda$ es un factor de ponderación para minimizar los dos objetivos en simultáneo, en este problema se define a $\lambda$ con el valor 0,5 .

Para la resolución del problema de flujo de potencia del sistema de distribución radial se utilizó el método iterativo conocido como "doble barrido" (Prasad, 2007). Debido a la naturaleza combinatoria del problema de optimización, del tipo NP-completo, se implementó el algoritmo heurístico conocido como recocido simulado (Kirkpatrick, 1983) para minimizar la función objetivo, permitiendo una buena exploración del espacio solución. Básicamente, en este algoritmo se proponen nuevas líneas a segmentar y distintos nodos donde realizar las inyecciones. A partir de esta nueva configuración se calcula el valor de la función objetivo y se repite este proceso iterativamente guardando la mejor solución encontrada en cada paso. Como una característica distintiva del algoritmo recocido simulado, se permite aceptar soluciones que aumenten el valor de la función objetivo para evitar quedar atrapado en un mínimo local.

Esta herramienta de diseño se aplicó a la red de estudio de 69 nodos de PG\&E, California, Estados Unidos (Figura 1), y a una red con datos de relevamiento propio, ubicada en la localidad de Bella Italia, provincia de Santa Fe (Figura 2 y 4 .A). En ambos casos se simuló la incorporación óptima de generadores, así como la ubicación más adecuada de las segmentaciones que 
delimitan las microrredes. En los casos estudiados se propuso evaluar la metodología con tres segmentaciones y tres inyecciones de potencia. Las potencias inyectadas a la red de 69 nodos fueron de 600, 900 y $1500 \mathrm{KW}$. Para la red de Bella Italia las potencias inyectadas fueron de 100, 70 y $30 \mathrm{KW}$. Los datos de la red de 69 nodos se pueden encontrar en (Baran, 1989) y los datos relevados de la red de Bella Italia se detallan en la tabla 1.

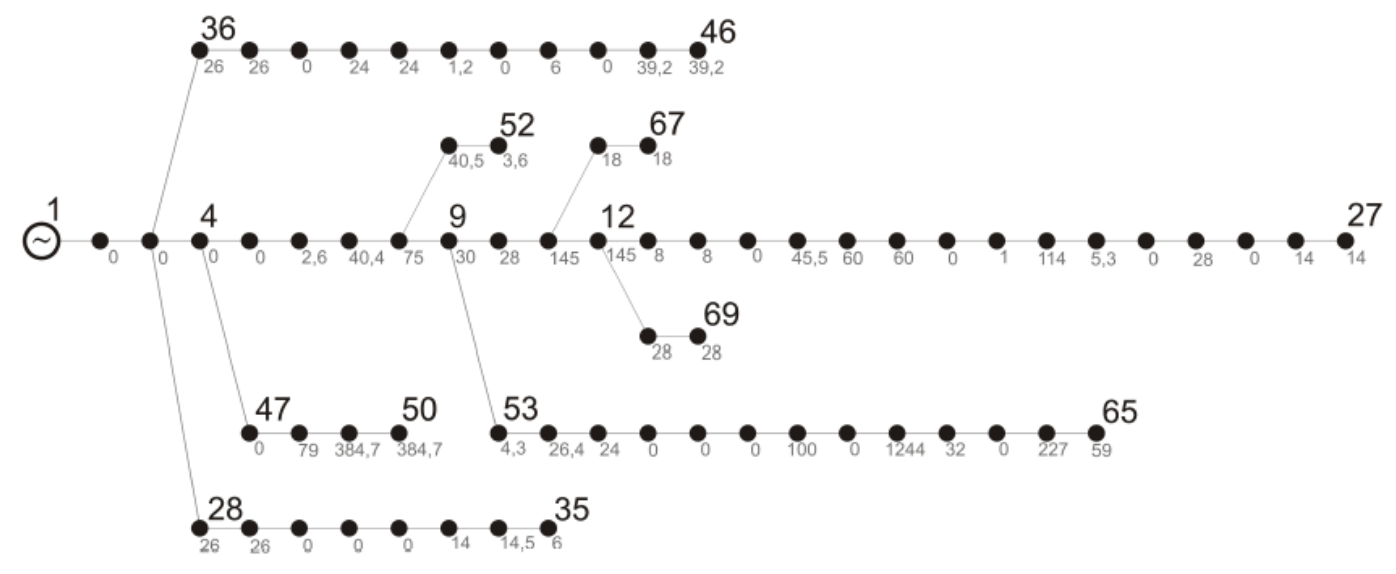

Figura 1. Topología de la red de 69 nodos.

En la figura 1 se muestra la estructura radial de la red de 69 nodos. El nodo 1 corresponde a la subestación y los demás son nodos de consumo. Debajo de cada nodo (en color gris) se indica la potencia activa demandada. Sobre algunos nodos (en color negro) se indica la numeración de los mismos.

\begin{tabular}{|r|c|c|r|r|r|r|}
\hline Línea & $\begin{array}{r}\text { Nodo } \\
\text { inicial }\end{array}$ & $\begin{array}{c}\text { Nodo } \\
\text { final }\end{array}$ & $\mathbf{R}(\mathbf{o h m})$ & $\mathbf{X}(\mathbf{o h m})$ & $\mathbf{P}(\mathbf{k W})$ & $\mathbf{Q}(\mathbf{k V A r})$ \\
\hline 1 & 1 & 2 & 0,673 & 0,073 & 32,91 & 20,39 \\
\hline 2 & 2 & 3 & 0,106 & 0,011 & 18,44 & 11,43 \\
\hline 3 & 3 & 4 & 0,274 & 0,029 & 34,83 & 21,59 \\
\hline 4 & 4 & 5 & 0,684 & 0,074 & 26,11 & 16,18 \\
\hline 5 & 5 & 6 & 0,302 & 0,032 & 24,88 & 15,42 \\
\hline 6 & 3 & 7 & 0,269 & 0,029 & 20,66 & 12,8 \\
\hline 7 & 7 & 8 & 0,802 & 0,087 & 21,13 & 13,09 \\
\hline 8 & 1 & 9 & 3,837 & 0,417 & 10,65 & 6,6 \\
\hline 9 & 9 & 10 & 0,448 & 0,048 & 17,33 & 10,74 \\
\hline 10 & 1 & 11 & 2,603 & 0,283 & 25,12 & 15,56 \\
\hline 11 & 11 & 12 & 0,044 & 0,004 & 1,28 & 0,79 \\
\hline 12 & 12 & 13 & 0,005 & 0,000 & 1,28 & 0,79 \\
\hline 13 & 13 & 14 & 0,095 & 0,010 & 38,12 & 23,62 \\
\hline 14 & 1 & 15 & 0,123 & 0,013 & 29,63 & 18,36 \\
\hline 15 & 15 & 16 & 0,153 & 0,016 & 25,12 & 15,56 \\
\hline
\end{tabular}

Tabla 1: Datos de la red de Bella Italia.

La tabla 1 expone los datos de la red de Bella Italia necesarios para resolver el flujo de potencia. La primera columna de la tabla refiere a la numeración de las líneas. Las columnas cuatro y cinco indican la impedancia de esas líneas. Las columnas dos y tres muestran la numeración de los nodos iniciales y finales respectivamente de cada línea. Y las columnas seis y siete son los valores de demanda activa y reactiva correspondientes al nodo terminal de la línea. En la figura 2 se observa la distribución de la red sobre una imagen aérea, en color amarillo se indican las líneas de transmisión y en círculos blancos los nodos numerados. En la figura 4A se muestra la topología de la red de Bella Italia. Al igual que en la red de 69 nodos, debajo de cada nodo en color gris se indica la potencia activa demandada y sobre algunos nodos en color negro se indica la numeración de los mismos. 


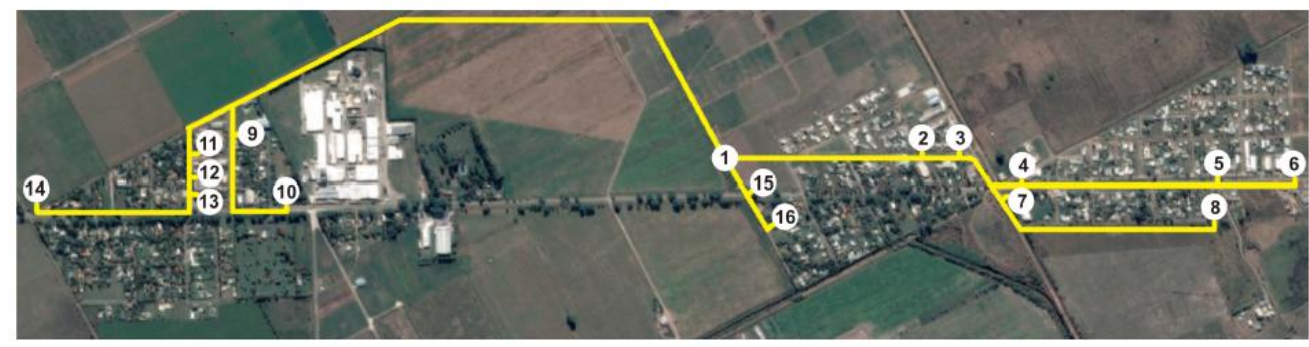

Figura 2. Imagen aérea de la red de Bella Italia.

\section{Resultados}

En la figura 3 se muestran los resultados obtenidos al aplicar la metodología propuesta a la red de 69 nodos. Con el mismo color se indican los nodos que forman parte de la misma microred luego de las segmentaciones. Las segmentaciones óptimas encontradas resultan en las líneas 11, 46 y 60, formando las tres microredes indicadas en color rojo, verde y azul respectivamente. En el recuadro de la figura 3 se indica el consumo total de cada microred, estos valores se obtienen sumando las demandas de potencia activa correspondientes al conjunto de nodos pertenecientes a una misma microred. También se muestra en esta imagen la mejor posición encontrada por el algoritmo para las inyecciones de potencia activa propuestas: los $600 \mathrm{KW}$ se inyectan en el nodo 16, los $900 \mathrm{KW}$ se inyectan en el nodo 49, y los $1500 \mathrm{KW}$ se inyectan en el nodo 61.

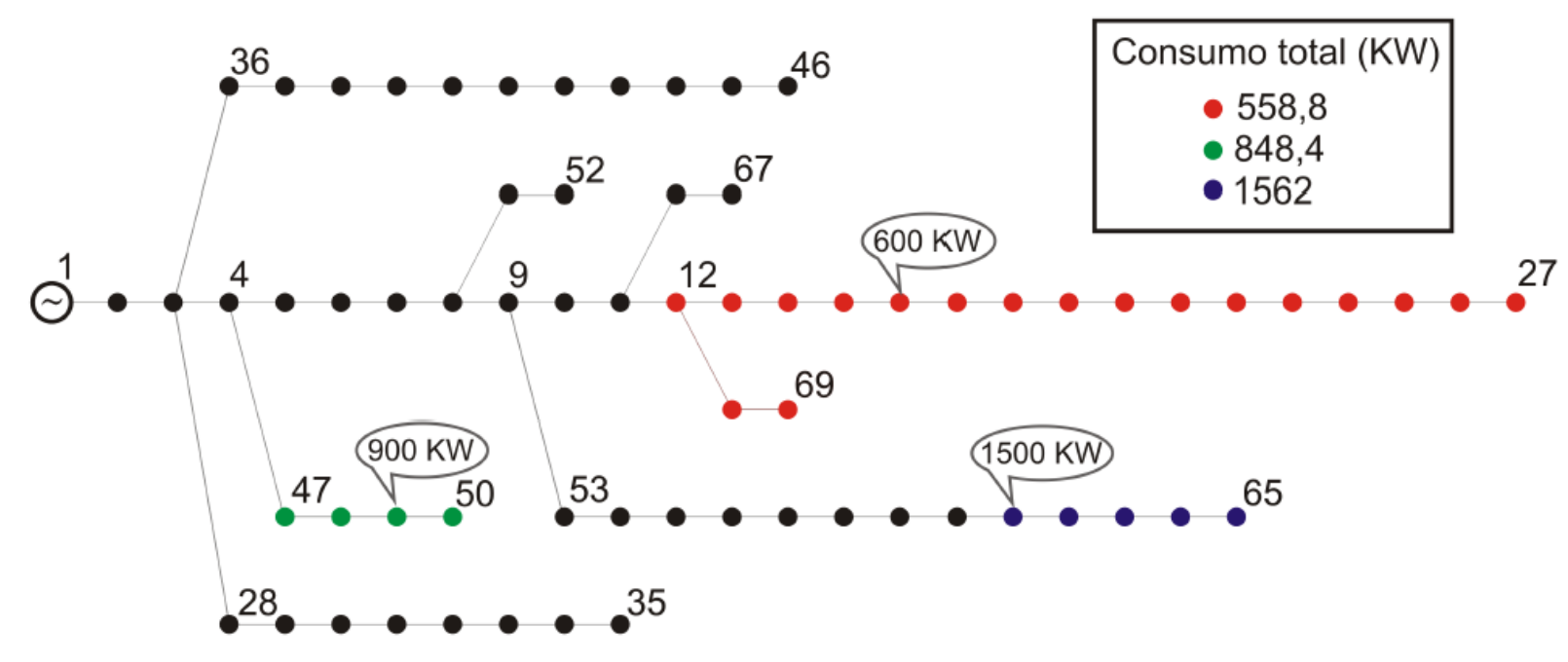

Figura 3. Inyección y segmentación óptima de la red de 69 nodos.

Los resultados de aplicar la metodología propuesta al segundo caso de estudio, la red de Bella Italia, se muestran en la figura 4B. Las mejores ubicaciones para las tres segmentaciones encontradas son las líneas 3, 8 y 10, delimitando las microredes indicadas en color rojo, azul y verde respectivamente. La mejor posición que halló el algoritmo para inyectar los $100 \mathrm{KW}$ propuestos de potencia activa es el nodo 5 , la mejor ubicación para inyectar los $30 \mathrm{KW}$ propuestos es el nodo 10 , y para inyectar los $70 \mathrm{KW}$ la mejor posición encontrada es el nodo 14. En el recuadro de la figura $4 \mathbf{B}$ se indican las potencias activas demandadas por el conjunto de nodos que conforman cada microred. 

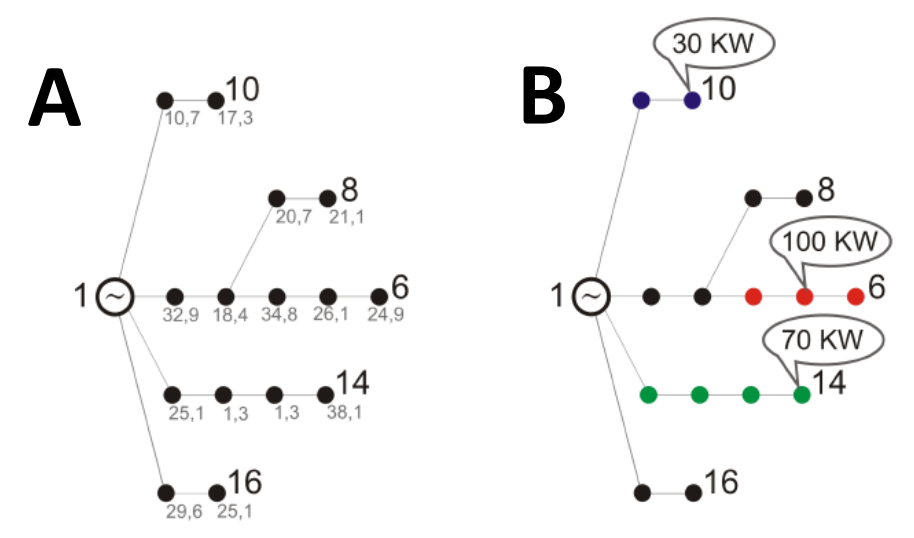
Consumo total $(\mathrm{KW})$
- 85,8
- 65,8
- 28

Figura 4. A. Topología de la red de Bella Italia. B. Inyección y segmentación óptima en la red de Bella Italia.

La inyección de potencia tiene mayor impacto en el funcionamiento global de la red. En la figura 5 se muestra la reducción de pérdidas de potencia en las redes estudiadas. Para la red de 69 nodos (Fig. 5A) la potencia disipada en la red sin inyección alcanza los $225 \mathrm{KW}$, mientras que con inyección las pérdidas alcanzan los $73 \mathrm{KW}$ (una reducción del 67,6\%). Algo similar ocurre con la red de Bella Italia (ver Fig. 5B), las pérdidas descienden de 3,50 KW a 1,54KW (una reducción del 55,9\%).
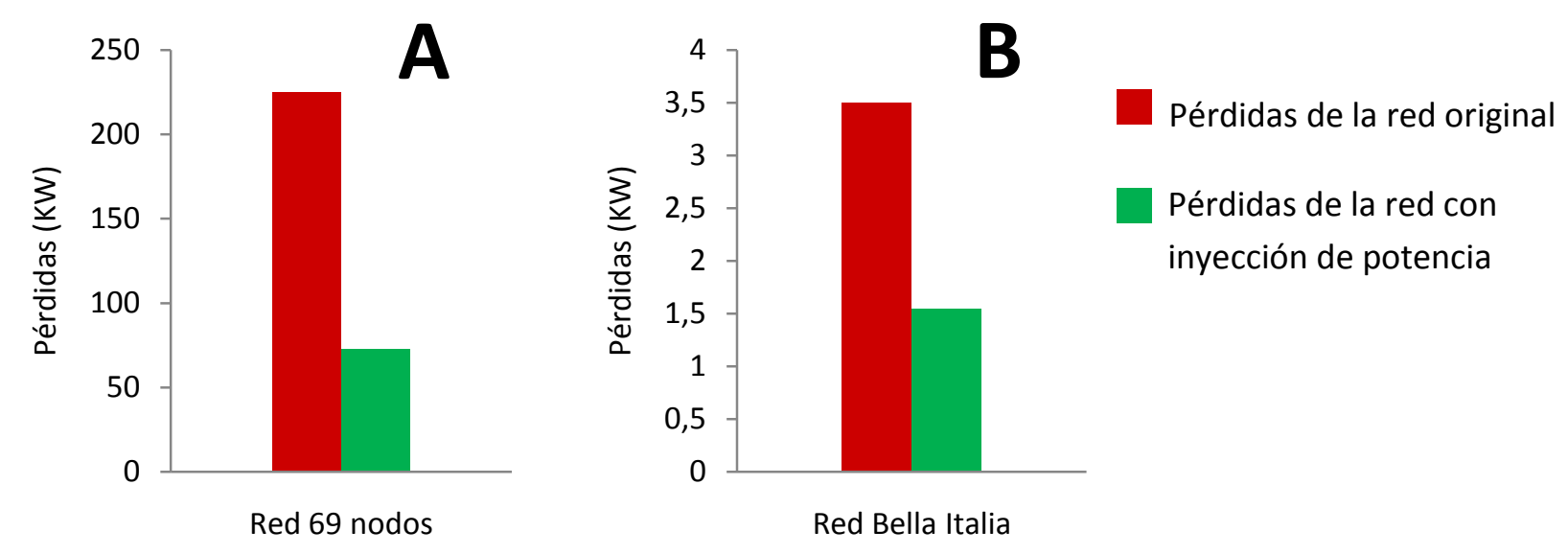

Figura 5. Reducción de las pérdidas de potencia en la red de 69 nodos (A) y en la red de Bella Italia (B).

\section{Conclusiones}

En este trabajo se propuso una metodología para el diseño óptimo de microredes basado en la sectorización de la red eléctrica. Con esta estrategia se logró que el conjunto de nodos que conforma cada microred tenga una demanda total del mismo orden que la potencia inyectada a esa microred. Este equilibrio entre las generaciones y las demandas se consiguió como resultado de los criterios empleados para la segmentación y la inyección de potencia, y ponen de manifiesto la relación dinámica e interdependiente de las dos variables de decisión (ubicación de inyecciones de potencia y ubicación de líneas a segmentar) al disminuir pérdidas y obtener autonomía potencial en las microredes. 
La optimización del presente trabajo considera el menor flujo de potencia entre microredes. Esto proporciona mayor autonomía, facilitando la gestión independiente de cada microred, aumentando la potencialidad de poder funcionar aislada ante una interrupción en el servicio eléctrico.

La herramienta propuesta reduce las pérdidas en las líneas mediante la generación distribuida. La inyección de potencia próxima a los puntos de consumo trae como consecuencia una menor transmisión de energía eléctrica por las líneas de la red, y una reducción de las pérdidas ya que dependen críticamente de la corriente en las líneas.

El método desarrollado permite diseñar microredes partiendo de redes radiales tradicionales, y no se focaliza en el mejor funcionamiento de una microred en particular, sino que busca el óptimo global con los recursos disponibles.

\section{Referencias}

Rau, N., Wan, Y. (1994). Optimum location of resources in distributed planning. IEEE Transactions on Power Systems, vol. 9, p. $2014-2020$.

Atwa, Y., El-Saadany, E., Salama, M., Seethapathy, R. (2010). Optimal Renewable Resources Mix for Distribution System Energy Loss Minimization. IEEE Transactions on Power Systems, vol. 25, p. 360-370.

Chowdhury, S., Crossley, P. (2009). Microgrids and Active Distribution Networks. London, U.K.: The Institution of Engineering and Technology.

Prommee, W., Ongsakul, W. (2011). Multiple Distributed Generation Placement in Microgrid System by Improved Reinitialized Social Structures Particle Swarm Optimization. European Transactions on Electrical Power, vol. 21, p. 489-504.

Beyza J., Yusta J. M., Correa G. J., Ruiz H. F. (2018). Vulnerability Assessment of a Large Electrical Grid by New Graph Theory Approach. IEEE Latin American Transactions, vol. 16, p. 527-535.

Arefifar, S., Mohamed, Y., El-Fouly, T. (2012). Supply-Adequacy-Based Optimal Construction of Microgrids in Smart Distribution Systems. IEEE Transactions on Smart Grid, vol. 3, p. 1491-1501.

Prasad K., Sahoo N. C., Chaturvedi A., Ranjan R. (2007). A Simple Approach for Branch Current Computation in Load Flow Analysis of Radial Distribution Systems. International Journal of Electrical Engineering Education, vol. 44, p. 49-63.

Kirkpatrick, S., Gelatt, J., Vecchi, M. (1983). Optimization by simulated annealing. Science, vol. 220, p. 671-680.

Baran, M. E., WU, F. F. (1989). Optimal Capacitor Placement on Radial Distribution System. IEEE Transactions on Power Delivery, vol. 4, p. $725-734$. 